\title{
X-RAY SPECTRA FROM HIGHLY IONIZED IRON AND NICKEL*
}

\author{
J. L. SCHWOB** and B.S. FRAENKEL
}

Laboratory of $X$-Rays and Far UV Spectroscopy, The Racah Institute of Physics, The Hebrew University, Jerusalem, Israel

$\mathrm{X}$-ray radiation is obtained from a point plasma produced by pinch effect Cohen et al. (1968) in a vacuum spark similar to that used by Lie and Elton (1971) with a $220 \mathrm{kA}$ peak current. The extremely dense and hot minute plasma appears at a distance of 0.5 to $1 \mathrm{~mm}$ from the anode tip and has maximum dimensions of a few microns.

The spectroscopic observations are performed by means of a focusing Cauchois spectrometer so as to avoid effects of fluctuations in the source position.

Spectra between 1 and $2 \AA$ of nearly completely stripped iron (Figure 1 ) and nickel were obtained. These spectra include optical transitions arising from a $2 p$ to 1 s electron jump in hydrogenic ions (FexxvI) and in helium-like ions (Fexxv and NixxvII) as well as satellite lines due to inner-shell transitions in lower ionization stage species from lithium-like down to fluorine-like ions. The plasma radiation is distinguished

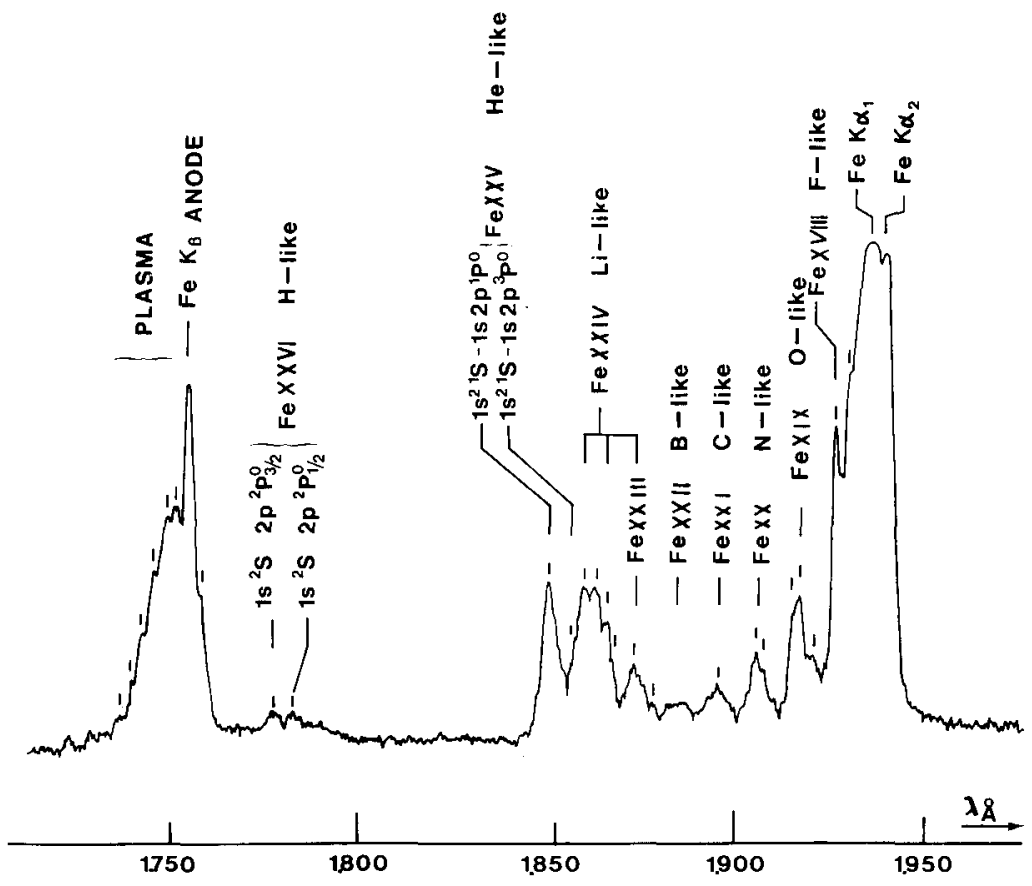

[Fig. 1. X-ray spectrum of highly ionized iron.

* Initial experiments were made at NASA Ames Research Center by BSF, on an NSF research fellowship during 1968-1970. The work presented here was facilitated in part by the Ford Foundation. ** On leave of absence from the Laboratoire des Hautes Pressions, CNRS - 92 Bellevue (France). 
from the anode characteristic radiation $\left(K \alpha_{1}, K \alpha_{2}\right.$ and $\left.K \beta\right)$ by means of a shadow technique using a tungsten wire placed between the source and the crystal.

The high spectral resolution achieved here, better than $0.3 \mathrm{X}$-unit, constitutes a substantial improvement relative to the previous observations on solar flares Neupert and Schwartz (1970), and on laboratory plasmas Lie and Elton (1970) concerning the Fe spectrum. This allowed us:

(1) to separate a number of line components;

(2) to identify a new line, attributed to the fluorine-like ion (FexvIII and Nixx) in good agreement with theoretical prediction of House (1969) (this line was masked by the $\mathrm{K} \alpha$ anode line in previous experiments Lie and Elton (1970) using a flat crystal);

(3) to discover a satellite feature emitted from the plasma also for the $\mathrm{K} \beta$ line; and

(4) finally to verify experimentally by the shadow method a slight shift towards the longer wavelength side of $\mathrm{K} \alpha$, as predicted by House (1969), which would be due to FeviII-X emission. This feature is not observed for the $\mathrm{K} \beta$ line.

The intensity ratio of the hydrogenic ion $L y-\alpha$ line and the helium-like ion resonant singlet in the case of $\mathrm{Fe}$, would lead in steady state coronal approximation to an electron temperature $T_{\mathrm{e}}$ of about $4 \mathrm{keV}$. In fact the short time duration of te dense plasma $\left(5 \times 10^{-8} \mathrm{~s}\right)$ does not allow the reaching of a steady state. We therefore have to expect a still higher electron temperature in our transient plasma, the preceding value representing only a lower limit value.

Clearly broad lines such as the helium-like ion singlet are easily distinguishable from lines of lesser width such as the fluorine-like ion line. The instrumental function is determined by ordinary X-ray lines. Thus, assuming thermal Doppler broadening, we obtain an ion temperature of the order of $30 \mathrm{keV}$.

The results are consistent with the $T_{\mathrm{e}}$ measurement by Lie and Elton (1970) from a continuum radiation method.

\section{References}

Cohen, L., Feldman, U., Swartz, M., and Underwood, J. H.: 1968, J. Opt. Soc. Am. 58, 843.

Lie, T. N. and Elton, R. C.: 1971, Phys. Rev. A3, 865.

Neupert, W. M. and Swartz, M.: 1970, Astrophys. J. 160, L189.

House, L. L.: 1969, Astrophys. J. Suppl. 18, 21.

\section{DISCUSSION}

J. Kistemaker: What is the electron density in your spark?

B. S. Fraenkel: The electron density is of the order $10^{20} \mathrm{~cm}^{-3}$.

F. Saris: First I would like to make a comment. In the field of atomic collisions one is studying $X$-ray production in ion-atom collisions by firing an ion beam through a gas or a thin film. In these experiments copious $X$-rays are observed from highly ionized particles. Secondly I would like to ask you to explain the shift towards longer wavelengths of the $\mathrm{K} \alpha \mathrm{X}$-ray lines and why does this not occur for the $\mathrm{K} \beta$ lines. In the above mentioned experiments one observes a shift to shorter wavelengths of the Fe K $\alpha$ lines (D. Burch et al., Phys. Rev. Letters 26 (1971), 1355).

B. S. Fraenkel: This lengthening, for Fe VIII to Fex, has been predicted by House, in Hartree-Fock calculations. This is only a very small amount, but it could be verified by our spectrograph. It is almost impossible to see this except with high resolution. Experimentally it does not show in the $\mathrm{K} \beta$. 
G. Mehlman-Balloffer: For such high density plasmas how do you explain such large differences between ionic and electronic temperatures?

$J$. L. Schwob: The electronic temperature given here is only a minimum value corresponding to a steady state, whereas our conditions are transient. Therefore a higher electronic temperature is to be expected.

H. Conrads: Did you determine the line profile? Is it Gaussian?

J. L. Schwob: We assume a Gaussian profile, we did however not determine it. The ion-ion collision time is extremely short relative to the life-time of the instability.

$H$. Conrads: Do you have any indication of a motion of the plasma-ball?

J. L. Schwob: None other than the short duration of the unstability before collapsing which is less than $5 \times 10^{-8} \mathrm{~s}$.

H. Conrads: From which direction did you observe the spectra?

J. L. Schwob: Perpendicular to the axis of the electrodes.

J. Kistemaker: What is the size of your plasma?

B.S. Fraenkel: The size is of the order of sphere with diameter of 1 to $3 \mu$. This can be shown by the pinhole camera photograph, and, specifically by the sharp edge of the image of the instability in the pinch.

$H$. -J. Kunze: In a rather similar device operating at a charging voltage of $10 \mathrm{kV}$ we measure average electron temperatures of $30 \mathrm{keV}$ using the foil absorption technique.

$J$. L. Schwob: This value agrees with our conclusions, which gave only a minimum electron temperature for a steady state, while we are in a transient regime, this implying higher temperatures. 